\title{
Computed tomography of the head for adult patients with minor head injury: are clinical decision rules a necessary evil?
}

\author{
Desmond Wei $\underline{T a n}^{1}$, MBBS, Annabelle Mei En Lim ${ }^{1}$, MBBS, Daniel Yuxuan $\underline{O n g}^{2}$, Li Lee Peng ${ }^{3,4}$, MBBS, FRCSEd,
} Yiong Huak $\underline{\mathrm{Chan}}^{5}$, PhD, Irwani Ibrahim ${ }^{3,4}$, FRSCEd, MPH, Win Sen $\underline{\text { Kuan }}^{3,4}$, MRCSEd, MCI

\begin{abstract}
INTRODUCTION This study aimed to evaluate compliance with and performance of the Canadian Computed Tomography Head Rule (CCHR), and its applicability to the Singapore adult population with minor head injury.

METHODS We conducted a retrospective study over six months of consecutive patients who presented to the adult emergency department (ED) with minor head injury. Data on predictor variables indicated in the CCHR was collected and compliance with the CCHR was assessed by comparing the recommendations for head computed tomography (CT) to its actual usage.

RESULTS In total, 349 patients satisfied the inclusion criteria. Common mechanisms of injury were falls (59.3\%), motor vehicle crashes (16.9\%) and assault (12.0\%). 249 (71.3\%) patients underwent head CT, yielding $42(12.0 \%)$ clinically significant findings. 1 (0.3\%) patient required neurosurgical intervention. According to the CCHR, head CT was recommended for 209 (59.9\%) patients. Compliance with the CCHR was 71.3\%. Among the noncompliant group, head CT was overperformed for $20.1 \%$ and underperformed for $8.6 \%$ of patients. Multivariate logistic regression analysis revealed that absence of retrograde amnesia (odds ratio [OR] 4.1,95\% confidence interval [Cl] 1.8-9.7) was associated with noncompliance to the CCHR. Factors associated with underperformance were absence of motor vehicle crashes as a mechanism of injury (OR 6.6, 95\% $\mathrm{Cl} 1.2-36.3$ ) and absence of headache (OR 10.8, 95\% $\mathrm{Cl} 1.3-87.4$ ).

CONCLUSION Compliance with the CCHR for adult patients with minor head injury remains low in the ED. A qualitative review of physicians' practices and patients' preferences may be carried out to evaluate reasons for noncompliance.
\end{abstract}

Keywords: decision support techniques, guideline adherence, head injuries

\section{INTRODUCTION}

Minor head injury (MHI) is a common condition seen in the emergency department (ED). ${ }^{(1)}$ Most authors have defined MHI as blunt injury to the head with alteration in consciousness, amnesia or disorientation in a patient who has a Glasgow Coma Scale (GCS) score of 13-15. ${ }^{(2)}$ While most patients with $\mathrm{MHI}$ do not require treatment, an estimated $6 \%-9 \%$ of patients have intracranial injuries and $0.4 \%-1 \%$ of patients require critical neurosurgical intervention. ${ }^{(3)}$ Computed tomography (CT) has proven to be an effective modality in early diagnosis and intervention of $\mathrm{MHI} .{ }^{(4)}$ However, fear of the dire consequences of delayed treatment subsequent to $\mathrm{MHI}$ has led to its liberal routine use. $^{(5)}$ The extensive use of CT for patients with $\mathrm{MHI}$ is not a costeffective strategy, as potentially life-threatening complications occur at low rates in these patients. In addition, concerns have been raised over unnecessary exposure of patients to ionising radiation, although the calculated projected cancer risk from such exposure remains controversial. ${ }^{(6)}$

To standardise and increase the efficiency of CT usage, independent clinical decision rules, such as the Canadian Computed Tomography Head Rule (CCHR), have been developed. ${ }^{(7)}$ The CCHR has been externally validated and was found to be highly sensitive for detecting intracranial injuries, but with variation in its specificity. ${ }^{(8)}$ Studies evaluating the performance of the CCHR have been performed mainly in American, European and Australian patients. ${ }^{(9-11)}$ There is currently limited literature evaluating the performance of $\mathrm{CCHR}$ in Asian populations, as they have different epidemiological and socioeconomic features as well as diverse healthcare systems. ${ }^{(12,13)}$ Although the CCHR was derived over a decade ago, adherence to these rules has been inconsistent, ranging from $12 \%$ to $57 \%$ in an international survey. ${ }^{(14)}$

The primary objective of the present study was to determine compliance with the CCHR for CT in patients with $\mathrm{MHI}$. The secondary objective was to evaluate the performance of the CCHR at a tertiary hospital ED in Singapore. We hypothesised that there would be low compliance with the $\mathrm{CCHR}$, resulting in unnecessary $\mathrm{CT}$ being performed for patients with $\mathrm{MHI}$.

\section{METHODS}

A retrospective study was conducted of consecutive patients presenting with acute $\mathrm{MHI}$ to the ED at National University Hospital (NUH), Singapore, over a six-month period, from 1 January 2013 to 30 June 2013. NUH, a 1,100-bed tertiary academic medical centre that receives about 130,000 adult attendances per year, has guidelines for the use of the CCHR

${ }^{1}$ Ministry of Health Holdings, ${ }^{2}$ Yong Loo Lin School of Medicine, National University of Singapore, ${ }^{3}$ Emergency Medicine Department, National University Hospital, ${ }^{4}$ Department of Surgery, ${ }^{5}$ Biostatistics Unit, Yong Loo Lin School of Medicine, National University of Singapore, Singapore

Correspondence: Dr Win Sen Kuan, Senior Consultant, Emergency Medicine Department, National University Hospital, 5 Lower Kent Ridge Road, Singapore 119074. win_sen_kuan@nuhs.edu.sg 
for patients presenting with $\mathrm{MHI}$ that aid in decision-making on whether to perform head CT for such patients. The study was approved by the National Healthcare Group's Domain Specific Review Board (2014/00615), which also granted waiver of consent.

Electronic medical records (ED Web) at the NUH ED were utilised for the selection of patients. To identify potential patients, the search for primary and secondary diagnoses was carried out using the International Classification of Diseases, Ninth Revision, Clinical Modification coding (ICD-9-CM), with diagnosis codes 959.01 (head injury, unspecified) and 850.0 (concussion with no loss of consciousness) and free-text searches, such as *head injury* and *concussion*. A further search of primary and secondary diagnoses, and review of every patient using keywords, such as *subdural*, *subarachnoid*, *epidural*, *extradural*, *intracranial**haemorrhage*, ${ }^{*}$ cerebral ${ }^{*}$ *contusion*, *intraparenchymal*, *brain**injury*, *fracture* AND *skull*/*frontal*/*parietal*/*temporal*/*occipital*, and *cerebral**oedema*, was also done to identify patients who were inadvertently missed during the initial searches.

Acute $\mathrm{MHI}$ was defined as a history of blunt trauma to the head within 24 hours of presentation to the ED and a GCS score of 13-15. Exclusion criteria included: (a) patients below 16 years of age; (b) obvious penetrating skull injury or depressed skull fracture; (c) unstable vital signs associated with major trauma; (d) presence of bleeding disorder (e.g. haemophilia) or use of oral anticoagulants; (e) patients returning for reassessment of the same head injury; (f) contraindications for CT (e.g. pregnancy); and (g) patients with neurological deficits. We ascertained the compliance and performance of the CCHR using additional entry criteria, such as witnessed loss of consciousness, disorientation or amnesia. ${ }^{(7)}$

Data was collected on demographics, mechanisms of injury, risk factors stated in the $\mathrm{CCHR}$, clinical symptoms and examination findings, antiplatelet agent usage, radiological findings on CT and neurosurgical interventions. Need for neurosurgical intervention was defined as requiring any of the following procedures within seven days of injury: craniotomy; elevation of skull fracture; intracranial pressure monitoring; or intubation for head injury. CT interpretations by radiologists were considered as the reference standard. Patients who did not undergo CT evaluation received follow-up of up to 14 days to determine re-attendances at the ED. A time frame of 14 days was used, as the presence of intracranial haemorrhage, if any, would have resulted in clinically significant manifestations in these patients by then.

CT findings considered as positive and clinically significant were: epidural haematoma, subdural haematoma of thickness $\geq 4 \mathrm{~mm}$, subarachnoid haemorrhage of thickness $>1 \mathrm{~mm}$, intracerebral haematoma, intraventricular haemorrhage, diffuse cerebral oedema, cerebral contusion of diameter $\geq 5 \mathrm{~mm}$, pneumocephalus and depressed skull fracture. Clinically insignificant brain injuries were focal subarachnoid haemorrhage, cerebral contusion of thickness $<5 \mathrm{~mm}$, subdural haematoma of thickness $<4 \mathrm{~mm}$, isolated pneumocephalus and closed depressed skull fracture not through the inner table. ${ }^{(7)}$ Signs and symptoms that were not documented in the physician and nursing notes were assumed to be absent. Data was collected by three investigators using a standardised data collection form and all completed forms were reviewed by the main investigator.

The main outcome we evaluated was compliance with (proportion of adherence to) the CCHR in the ED. This was performed by comparing recommendations for $\mathrm{CT}$ according to the CCHR criteria with the actual usage of CT. Noncompliance was further stratified into: (a) 'underperformed', where CT was not done despite the CCHR recommendation; or (b) 'overperformed', where CT was done despite the absence of the CCHR recommendation. The secondary outcome was performance of the $\mathrm{CCHR}$, as ascertained by using sensitivity, specificity, predictive values and likelihood ratios.

Categorical variables were reported as frequency and percentage values. Continuous variables were reported as median (interquartile range). All data was populated in a Microsoft Excel spreadsheet (Microsoft Corp, Redmond, WA, USA). Upon completion of data collection electronically, the charts were reviewed for missing or duplicated data and verified. The data was then exported to Stata 14 (StataCorp LP, College Station, TX, USA) for statistical analyses. Differences in categorical variables between the patient groups that were compliant and noncompliant with the CCHR recommendations were compared using chi-square test or Fisher's exact test. Highly skewed continuous outcomes were analysed using the Mann-Whitney $U$ test. Multivariate logistic regression analysis was performed to determine the characteristic differences between the two groups using odds ratio (OR) and 95\% confidence intervals (Cls). A stepwise approach was used while including only variables with $p<0.10$ into the model. A subgroup analysis was planned, with the hypothesis that those who were on antiplatelet agents were more likely to have had CT overperformed. Statistical significance was set at $\mathrm{p}<0.05$.

\section{RESULTS}

A total of 935 patients presented to the ED during the six-month period for traumatic head injury and 349 satisfied the CCHR entry criteria (Fig. 1). Compliance with the CCHR recommendations was found to be $71.3 \%$ for our patients. Among the 349 patients who satisfied the CCHR criteria, CT was not performed for 30 (8.6\%) patients despite the CCHR recommendation (underperformed), while for $70(20.1 \%)$ patients, CT was performed despite the absence of the CCHR recommendation (overperformed). Head CT was performed for 249 (71.3\%) patients and $42(12.0 \%)$ patients had clinically significant positive findings (Tables I and II). Among these 42 patients, only $1(0.3 \%)$ patient required neurosurgical intervention. $21(6.0 \%)$ patients had return visits to the ED for complaints related to the initial head injury, but none required neurosurgical intervention.

The only death occurred in the compliant group, in which the patient deteriorated after CT and required endotracheal intubation. In view of the comorbid conditions in this patient, a 'do not actively resuscitate' order was acquired and terminal extubation was performed owing to the poor prognosis of severe subdural haematoma. 


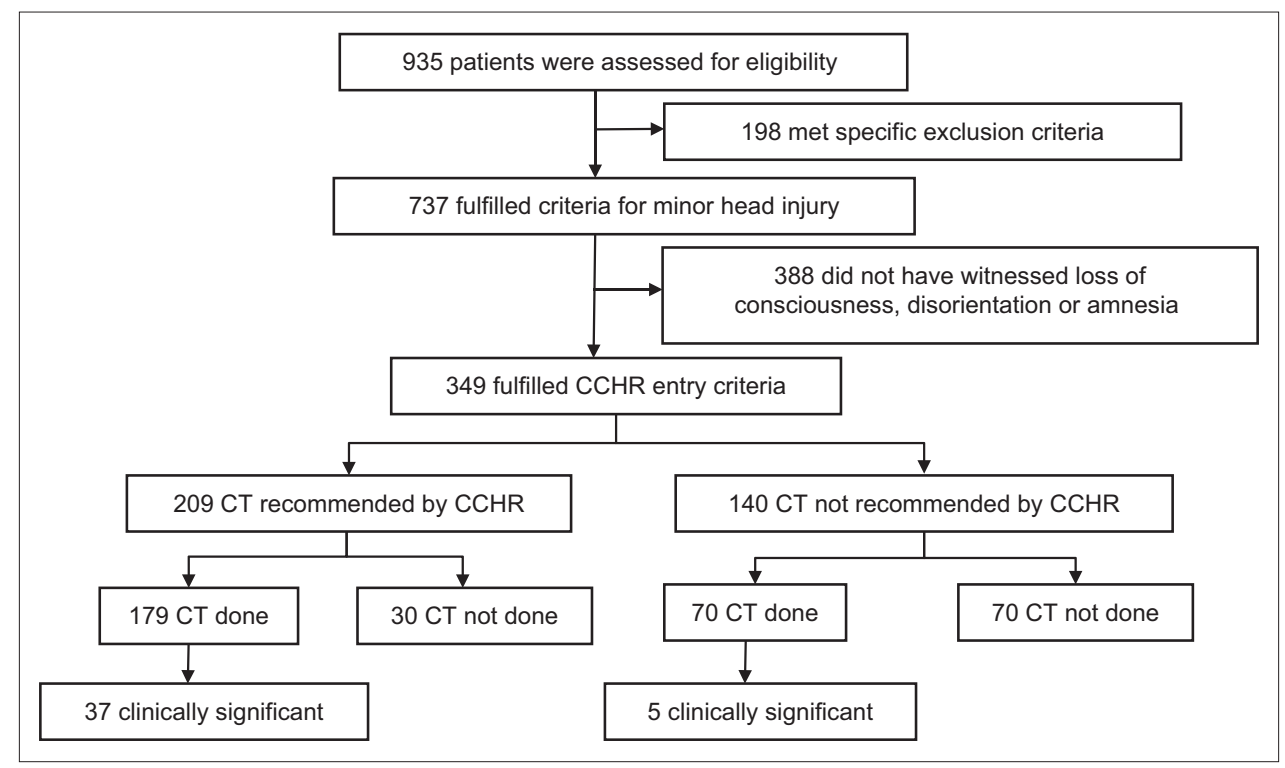

Fig. 1 Flowchart shows the assessment of patients who presented to the emergency department with traumatic head injury, based on the CCHR entry criteria. Noncompliance was defined as CT being underperformed or overperformed. CCHR: Canadian Computed Tomography Head Rule; CT: computed tomography.

The diagnostic performance of CCHR was evaluated (Table III). The likelihood ratios for positive and negative CT tests for clinically significant head injuries were $1.57(95 \% \mathrm{Cl}$ 1.35-1.82) and 0.27 (95\% Cl 0.12-0.62), respectively. Among 30 patients for whom CT was underperformed, two returned to the ED for the same head injury complaint, but neither required any neurosurgical intervention despite one patient having a CT scan that showed non-clinically significant cerebral contusion and subarachnoid haemorrhage. Among 70 patients for whom CT was overperformed, 8 (11.4\%) patients had positive CT findings, which were clinically significant for five patients although none of them underwent any neurosurgical intervention.

Multivariate logistic regression analysis revealed that the absence of retrograde amnesia was associated with noncompliance with the CCHR (OR 4.1, 95\% Cl 1.8-9.7). Factors associated with underperformance included the absence of motor vehicle crashes as a mechanism of injury (OR 6.6, $95 \% \mathrm{Cl} 1.2-36.3$ ) and absence of headache (OR 10.8, 95\% Cl 1.3-87.4) (Table IV). No significant factors were identified for the overperformance of CT.

A planned subgroup analysis was performed based on the hypothesis that physicians were more likely to order head CT for patients on antiplatelet therapy. Among 36 patients who were receiving aspirin and/or clopidogrel either alone or together, CT was overperformed for $4(11.1 \%)$ patients. Comparatively, CT was overperformed for 66/313 (21.1\%) patients among those who were not on antiplatelet therapy $(p=0.16)$.

\section{DISCUSSION}

In 2013, the Cost Effective Care Task Force of the American College of Emergency Physicians recommended the avoidance of head CT in ED patients with MHI who are considered low risk based on validated decision rules as one of five recommendations it made in the 'Choosing Wisely' campaign. ${ }^{(15)}$ In the present study, we sought to evaluate the compliance with the CCHR recommendations of patients presenting with $\mathrm{MHI}$ to our $\mathrm{ED}$, where physicians had been trained on the use of the CCHR to guide decision-making on performing CT. The study confirmed our original hypothesis that there was low compliance (71.3\%) with the CCHR recommendations despite its awareness and acceptance, which was similar to previous studies that reported compliance rates in the range of $60 \%-80 \%{ }^{(6,16)}$ An additional $70(20.1 \%)$ CT scans were performed by physicians despite not being recommended by the $\mathrm{CCHR}$, which was again similar to previous studies that reported avoidable CT being performed for $20 \%-40 \%$ of patients with $\mathrm{MHI} .{ }^{(6,17)}$ At the same time, a multicentre, cluster randomised trial conducted across 12 sites in Canada showed a paradoxical increase in $\mathrm{CT}$ performed $(76.2 \%)$ after the implementation of the $\mathrm{CCHR}$ for patients with $\mathrm{MHI},{ }^{(17)}$ perhaps suggesting that high compliance does not always equate with more efficient utility.

In our study, about one-fifth of patients with MHI could have averted the extra costs and radiation exposure associated with $\mathrm{CT}$, which was done despite not being indicated by the CCHR. Decision-making with regard to conducting CT for patients with $\mathrm{MHI}$ and its overperformance is influenced by both physician and patient factors, as the tendency for physicians and patients to overestimate the probability of finding a serious injury on CT has been reported to be around two times and six times, respectively. ${ }^{(18)}$ Results of the multivariate logistic regression analysis in our study suggest a possibility that certain variables that could have been associated with decision-making on performing $\mathrm{CT}$, such as individual perceptions and attitudes of physicians and patients, may not have been included in the analysis. A previous qualitative study identified six domains from the Theoretical Domains Framework that might pose as barriers for the use of the CCHR, namely: beliefs about consequences; beliefs about capabilities; behavioural regulation; memory, attention, and 
Table I. Characteristics of patients with minor head injury $(n=349)$.

\begin{tabular}{|c|c|c|c|}
\hline Characteristic & No. (\%) & Characteristic & No. (\%) \\
\hline Age $(y r)^{*}$ & $48(30-68)$ & Sports & $18(5.2)$ \\
\hline Male gender & $218(62.5)$ & Other & $1(0.3)$ \\
\hline Ethnicity & & Presence of $\mathrm{CCHR}$ variables & \\
\hline Chinese & $215(61.6)$ & GCS score $<15$ ( $2 \mathrm{hr}$ after injury) & $58(16.6)$ \\
\hline Malay & $64(18.3)$ & Signs of basal skull fracture & $3(0.9)$ \\
\hline Indian & $39(11.2)$ & Suspected open skull fracture & $9(2.6)$ \\
\hline Other & $31(8.9)$ & Vomiting $>1$ episode & $31(8.9)$ \\
\hline GCS score & & Age $\geq 65 \mathrm{yr}$ & $104(29.8)$ \\
\hline 13 & $19(5.4)$ & Retrograde amnesia $>30 \mathrm{~min}$ & $65(18.6)$ \\
\hline 14 & $39(11.2)$ & Dangerous mechanism of injury & $29(8.3)$ \\
\hline 15 & $291(83.4)$ & Disposition & \\
\hline Antiplatelet therapy & & Discharged from ED & $116(33.2)$ \\
\hline Aspirin & $25(7.2)$ & ED observation unit & $81(23.2)$ \\
\hline Clopidogrel & $6(1.7)$ & Admitted to inpatient ward & $152(43.6)$ \\
\hline Aspirin and clopidogrel & $5(1.4)$ & Revisited ED & $21(6.0)$ \\
\hline Mechanism of injury & & CT of head & \\
\hline Fall & 207 (59.3) & Recommended $^{+}$ & 209 (59.9) \\
\hline From standing or sitting position & 178 & Recommended but not performed & $30(8.6)$ \\
\hline From elevation $<1 \mathrm{~m}$ or 5 steps & 17 & Performed & $249(71.3)$ \\
\hline From elevation $<3$ m or 15 steps & 8 & Performed but not recommended ${ }^{+}$ & $70(20.1)$ \\
\hline From elevation $>3$ m or $>15$ steps & 4 & Positive CT & $50(14.3)$ \\
\hline Motor vehicle crash & $59(16.9)$ & Clinically significant positive CT & $42(12.0)$ \\
\hline Cyclist/motorcyclist & 30 & Neurosurgical intervention & $1(0.3)$ \\
\hline Car occupant & 21 & Death secondary to head injury & $1(0.3)$ \\
\hline Pedestrian & 8 & & \\
\hline Assault & $42(12.0)$ & & \\
\hline External environment & $22(6.3)$ & & \\
\hline Head struck by object & 20 & & \\
\hline Head hit object head-on & 2 & & \\
\hline
\end{tabular}

*Data presented as median (interquartile range). †Based on the Canadian Computed Tomography Head Rule. CT: computed tomography; ED: emergency department; GCS: Glasgow Coma Scale

decision processes; environment context and resources; and social influences. ${ }^{(19)}$ Future prospective studies could include these factors for a more robust analysis.

Studies have shown that physicians are often either unaware of the clinical decision rules or ignore them in clinical practice. ${ }^{(6,20)}$ For example, awareness of the CCHR was $31 \%$ in the United States, which has a low CCHR usage rate of $12 \%$, while in Australasia and the United Kingdom, despite a higher awareness of the CCHR at $82 \%$ and $66 \%$, the usage rate was only about one-third and one-fifth, respectively. ${ }^{(14)}$ These findings suggest that awareness of clinical decision rules does not necessarily imply acceptance of them in daily clinical practice, thereby leading to low compliance. Along similar lines, in our ED, although awareness of the CCHR was $100 \%$ among senior faculty, compliance was only $71.3 \%$.

In our study, CT that was ordered for patients in the overperformed group (20.1\%) detected clinically significant findings in an additional five patients (i.e. $11.9 \%$ of 42 clinically significant CT findings) that would otherwise have been missed had the CCHR been strictly adhered to. Some physicians may justify such use of additional CT for a high-stakes diagnosis of a significant intracranial injury, even though the treatment is
Table II. Patients with positive findings on head CT scans.

\begin{tabular}{|lll|}
\hline Finding & \multicolumn{2}{c|}{ No. (\%) } \\
\cline { 2 - 3 } & $\begin{array}{l}\text { Positive } \mathbf{C T}^{*} \\
(\mathbf{n}=\mathbf{5 0})\end{array}$ & $\begin{array}{l}\text { Clinically significant } \\
\text { positive } \mathbf{C T}^{*}(\mathbf{n}=\mathbf{4 2})\end{array}$ \\
\hline Haematoma & & \\
\hline Epidural & $5(10.0)$ & $5(11.9)$ \\
\hline Subdural & $21(42.0)$ & $14(33.3)$ \\
\hline Intracerebral & $6(12.0)$ & $6(14.3)$ \\
\hline Haemorrhage & & \\
\hline Subarachnoid & $29(58.0)$ & $27(64.3)$ \\
\hline Intraventricular & $0(0)$ & $0(0)$ \\
\hline Diffuse cerebral oedema & $0(0)$ & $0(0)$ \\
\hline Cerebral contusion & $13(26.0)$ & $10(23.8)$ \\
\hline Pneumocephalus & $1(2.0)$ & $1(2.4)$ \\
\hline Depressed skull fracture & $10(20.0)$ & $10(23.8)$ \\
\hline
\end{tabular}

*Some patients had more than one lesion. CT: computed tomography

nonsurgical. Conversely, patients in the underperformed group who did not sustain a motor vehicle crash (OR 6.6) or have a headache (OR 10.8) were less likely to have CT done despite the CCHR recommendations. It is possible that knowledge of 
Table III. Diagnostic performance of the CCHR among patients with minor head injury.

\begin{tabular}{|lllllll|}
\hline CCHR recommendation & \multicolumn{2}{c}{ No. } & & & Likelihood ratios (95\% CI) \\
\cline { 2 - 4 } \cline { 6 - 7 } & Total $(\mathbf{n}=\mathbf{3 4 9})$ & $\begin{array}{l}\text { Clinically significant } \\
\text { injury }(\mathbf{n}=\mathbf{4 2})\end{array}$ & $\begin{array}{l}\text { No clinically significant } \\
\text { injury }(\mathbf{n}=\mathbf{3 0 7})\end{array}$ & Positive & Negative \\
\hline CT recommended & 209 & 37 & 172 & $1.57(1.35-1.82)$ & $0.27(0.12-0.62)$ \\
\hline CT not recommended & 140 & 5 & 135 & & \\
\hline
\end{tabular}

Sensitivity $88.1 \%$ (74.4\%-96.0\%), specificity $44.0 \%$ (38.3\%-49.7\%), positive predictive value $17.7 \%$ (12.8\%-23.6\%), negative predictive value $96.4 \%$ (91.9\%-98.8\%). CCHR: Canadian Computed Tomography Head Rule; Cl: confidence interval; CT: computed tomography

Table IV. Significant factors associated with underperformance of computed tomography.

\begin{tabular}{|c|c|c|c|c|}
\hline Variable & $\begin{array}{l}\text { Univariate OR } \\
(95 \% \mathrm{CI})\end{array}$ & p-value & $\begin{array}{l}\text { Adjusted OR } \\
(95 \% \mathrm{CI})\end{array}$ & p-value \\
\hline Absence of headache & $12.5(1.5-104.3)$ & 0.020 & $10.8(1.3-87.4)$ & 0.026 \\
\hline
\end{tabular}

$\mathrm{Cl}$ : confidence interval; OR: odds ratio

other clinical decision rules, such as the New Orleans Criteria, that include severe headache as an indication for CT may sway a physician's decision-making to order CT for a patient with MHI. ${ }^{(21)}$

Good communication between physicians and patients could help to ensure that the risks and benefits of CT are properly discussed, and that patients remain adequately monitored if scans are not performed. This aligns well with the objectives of the 'Choosing Wisely' campaign, which aims to promote conversation between physicians and patients while lessening the overuse of tests and supporting patients in making effective care choices. ${ }^{(15)}$ Clinical decision rules such as the $\mathrm{CCHR}$ remain necessary to everyday clinical practice as adjuncts in the decisionmaking process. Prudence in the use of the CCHR, perhaps in combination with several other validated rules, may yield a much lower proportion of false negative and possibly false positive results for patients with $\mathrm{MHI}$.

The CCHR has been externally validated, with some studies reporting high sensitivity. ${ }^{(8)}$ However, variability still exists, with some studies reporting less than $95 \%$ sensitivity. ${ }^{(12)}$ In our study, the CCHR had a sensitivity of only $88.1 \%$, which was highlighted by the non-negligible number of $5(7.1 \%)$ patients out of the 70 overperformed patients for whom there were clinically significant positive findings on CT. We surmise that the lack of consistently high sensitivities reported by other centres for the CCHR may have caused physicians who have a low tolerance for missed potential life-threatening pathologies to be hesitant to solely rely on the CCHR and instead choose to use their judgement while assessing patients. The medicolegal implications when such conditions are missed may also result in the practice of defensive medicine and assurance behaviour, which has been shown to be the highest among emergency physicians when compared to other specialties. ${ }^{(22)}$

There were several limitations to this study. First, being a single-centre study, the number of patients was relatively small in comparison with the CCHR derivation cohorts. Second, missing data due to the study's retrospective design, especially that on symptoms and signs, may have resulted in bias that may have affected decision-making on whether to perform CT for patients with $\mathrm{MHI}$. Third, it is possible that patients with multiple injuries, including head injury without primary or secondary diagnoses of
MHI, may have been inadvertently missed during the curation of patients. Fourth, our ED is located in a hospital that is designated locally as a trauma centre. Ambulances may preferentially send more severe cases to our ED, creating a biased representation of severity. This could possibly account for the higher prevalence of clinically significant findings seen on head CT in our cohort $(42 / 349,12.0 \%)$ when compared with other studies whose findings ranged from $3.5 \%$ to $8.1 \% .^{(7,9-11)} \mathrm{Fifth}$, we recognise that given the different healthcare practices and settings, our study results may not be generalisable to all populations, especially to areas with a higher incidence of severe traumatic brain injury or with different policies regarding healthcare reimbursements. Sixth, due to the nature of our study design, we cannot exclude the possibility that unreported events occurred following discharge of patients from our ED, although attempts were made to follow up on unscheduled return visits to other EDs or admissions to other restructured hospitals through the nationwide electronic medical records for up to 14 days.

In conclusion, compliance with the CCHR for adult patients with $\mathrm{MHI}$ was low in this ED in Singapore. The CCHR remains a useful adjunct that aids in the decision-making process during the evaluation of patients with MHI. A qualitative review of physicians' practices and patients' preferences may be carried out to evaluate the reasons for noncompliance with the $\mathrm{CCHR}$.

\section{REFERENCES}

1. Cassidy JD, Carroll LJ, Peloso PM, et al; WHO Collaborating Centre Task Force on Mild Traumatic Brain Injury. Incidence, risk factors and prevention of mild traumatic brain injury: results of the WHO Collaborating Centre Task Force on Mild Traumatic Brain Injury. J Rehabil Med 2004; 43(suppl):28-60.

2. Levin HS, Diaz-Arrastia RR. Diagnosis, prognosis, and clinical management of mild traumatic brain injury. Lancet Neurol 2015; 14:506-17.

3. af Geijerstam JL, Britton M. Mild head injury - mortality and complication rate: meta-analysis of findings in a systematic literature review. Acta Neurochir (Wien) 2003; 145:843-50.

4. Klement W, Wilk S, Michalowski W, et al. Predicting the need for CT imaging in children with minor head injury using an ensemble of Naive Bayes classifiers. Artif Intell Med 2012; 54:163-70.

5. Smits M, Dippel DW, Nederkoorn PJ, et al. Minor head injury: CT-based strategies for management-a cost-effectiveness analysis. Radiology 2010; 254:532-40.

6. Melnick ER, Szlezak CM, Bentley SK, et al. CT overuse for mild traumatic brain injury. Jt Comm J Qual Patient Saf 2012; 38:483-9.

7. Stiell IG, Wells GA, Vandemheen K, et al. The Canadian CT Head Rule for patients with minor head injury. Lancet 2001; 357:1391-6. 
8. Stein SC, Fabbri A, Servadei F, Glick HA. A critical comparison of clinical decision instruments for computed tomographic scanning in mild closed traumatic brain injury in adolescents and adults. Ann Emerg Med 2009; 53:180-8.

9. Papa L, Stiell IG, Clement CM, et al. Performance of the Canadian CT Head Rule and the New Orleans Criteria for predicting any traumatic intracranial injury on computed tomography in a United States Level I trauma center. Acad Emerg Med 2012; 19:2-10.

10. Smits M, Dippel DW, de Haan GG, et al. External validation of the Canadian CT Head Rule and the New Orleans Criteria for CT scanning in patients with minor head injury. JAMA 2005; 294:1519-25.

11. Rosengren D, Rothwell S, Brown AF, Chu K. The application of North American CT scan criteria to an Australian population with minor head injury. Emerg Med Australas 2004; 16:195-200.

12. Ro YS, Shin SD, Holmes JF, et al; Traumatic Brain Injury Research Network of Korea (TBI Network). Comparison of clinical performance of cranial computed tomography rules in patients with minor head injury: a multicenter prospective study. Acad Emerg Med 2011; 18:597-604.

13. Lo WS, Shih YN, Leung CS, et al. A retrospective study of patients with minor head injury to compare the Canadian CT Head Rule and the New Orleans Criteria. Hong Kong J Emerg Med 2016; 23:25-33.

14. Eagles D, Stiell IG, Clement CM, et al. International survey of emergency physicians' awareness and use of the Canadian Cervical-Spine Rule anc the Canadian Computed Tomography Head Rule. Acad Emerg Med 2008; 15:1256-61.
15. American College of Emergency Physicians. Choosing Wisely. Five things physicians and patients should question. Available at: http://www. choosingwisely.org/wp-content/uploads/2015/02/ACEP-Choosing-Wisely-List. pdf. Accessed May 17, 2016.

16. Korley FK, Morton MJ, Hill PM, et al. Agreement between routine emergency department care and clinical decision support recommended care in patients evaluated for mild traumatic brain injury. Acad Emerg Med 2013; 20:463-9.

17. Stiell IG, Clement CM, Grimshaw JM, et al. A prospective cluster-randomized trial to implement the Canadian CT Head Rule in emergency departments. CMAJ 2010; 182:1527-32.

18. Quass J, Derrick B, Mitrani L, et al. Survey of patient and physician influences and decision-making regarding $\mathrm{CT}$ utilization for minor head injury. Injury 2014; 45:1503-8.

19. Curran JA, Brehaut J, Patey AM, et al. Understanding the Canadian adult CT head rule trial: use of the theoretical domains framework for process evaluation. Implement Sci 2013; 8:25.

20. Heskestad B, Baardsen R, Helseth E, Ingebrigtsen T. Guideline compliance in management of minimal, mild, and moderate head injury: high frequency of noncompliance among individual physicians despite strong guideline support from clinical leaders. J Trauma 2008; 65:1309-13.

21. Haydel MJ, Preston CA, Mills TJ, et al. Indications for computed tomography in patients with minor head injury. N Engl J Med 2000; 343:100-5.

22. Studdert DM, Mello MM, Sage WM, et al. Defensive medicine among highrisk specialist physicians in a volatile malpractice environment. JAMA 2005; 293:2609-17. 